\title{
Serum Fetuin-A Levels Related with Microalbuminuria in Diet-Induced Obese Rats
}

\author{
Yanyan Li, Xiaodong Sun, and Yerong Yu \\ Department of Endocrinology and Metabolism, West China Hospital, Sichuan University, Chengdu, Sichuan 610041, China \\ Correspondence should be addressed to Yerong Yu; yerongyu@scu.edu.cn
}

Received 20 August 2012; Revised 29 November 2012; Accepted 4 December 2012

Academic Editor: Sharad Rastogi

Copyright (C) 2013 Yanyan Li et al. This is an open access article distributed under the Creative Commons Attribution License, which permits unrestricted use, distribution, and reproduction in any medium, provided the original work is properly cited.

\begin{abstract}
The aim of the study was to investigate the association between elevated serum fetuin-A and increased urine albumin excretion in obese rats, and whether increased urine albumin excretion was modified by improving hepatic steatosis and lipid metabolism disorder. Male Wistar rats 4 weeks in age were randomly divided into three groups and fed with normal chow (control group), high-fat chow (obesity group), or high-fat chow plus fenofibrate (fenofibrate group). After 24 weeks, both body weight and visceral fat/body weight ratio in obese rats were higher than in controls. A difference in serology markers and pathology associated with hepatic steatosis was also found among the three groups. Serum fetuin-A and the expression of NF- $\kappa$ B in the liver were increased, while serum adiponectin was decreased in obese rats in comparison to controls $(P<0.01)$. Urinary albumin/creatinine ratio (ACR) was increased in the obesity group compared to controls $(P<0.01)$. The fenofibrate intervention reduced serum fetuin-A and NF$\kappa \mathrm{B}$ expression in the liver and increased serum adiponectin compared to obese rats and was accompanied by decrease in ACR. A positive correlation was found between ACR and fetuin-A $(r=0.602, P<0.01)$, and a negative correlation was found between ACR and adiponectin $(r=-0.635, P<0.01)$. We conclude that elevated fetuin-A levels are associated with microalbuminuria in obese rats, and abnormal albuminuria is reversible by improving hepatic steatosis.
\end{abstract}

\section{Introduction}

In recent decades, changes in lifestyle and diet have led to an increased frequency in overweight and obese individuals. Obesity has become one of the most urgent public health problems and causes a major threat to human health worldwide. Obesity is associated with chronic diseases such as nonalcoholic fatty liver disease (NAFLD) and chronic kidney disease (CKD), among others. Growing recognition of the importance of obesity-related NAFLD and obesity-related CKD has provoked interest in the potential link between NAFLD and CKD. There is increasing evidence that NAFLD is associated with an increased prevalence and incidence of CKD. This association seems to be independent of obesity, hypertension, diabetes, and other possibly confounding factors $[1,2]$. Moreover, prediabetic and newly diagnosed diabetic patients with NAFLD had higher prevalence rates of microalbuminuria than those without NAFLD [3]. However, the mechanistic relationship between NAFLD and CKD remains unknown.
Fetuin-A is an abundant serum protein, that is, predominantly synthesized and secreted by the liver. Higher serum fetuin-A levels have been found in diet-induced obesity, NAFLD and metabolic syndrome (MetS) [4-6]. Stefan and Haring reported that fatty liver might play an important role in the pathogenesis of the metabolic diseases type-2 diabetes mellitus (T2DM) and cardiovascular disease, and fetuin-A is directly involved in the pathogenesis of these diseases. However, recent novel findings revealed that under certain conditions fatty liver is not associated with metabolic disorders; the terms "metabolically benign" has been used to describe this state [7]. Adiponectin, a plasma protein primarily secreted by adipose tissue, was found to be decreased in NAFLD [8]. Both human fetuin-A and adiponectin genes reside on chromosome 3q27, which has been mapped as a T2DM and MetS susceptibility locus $[9,10]$. Hennige and colleagues demonstrated that the liver-derived fetuin-A induced lowgrade inflammation and repressed adiponectin production in animals and humans [11]. Moreover, adiponectin is a key regulator of albuminuria and is inversely related to 
albuminuria [12]. Low-grade inflammation, one of the characteristics of chronic kidney disease (CKD), is also associated with albuminuria. From this information, we hypothesized that high serum fetuin-A levels may be associated with increased urine albumin excretion. One study reported that serum fetuin-A level was elevated in subjects with fat accumulation in the liver, and a decrease in liver fat was accompanied by a decrease in serum fetuin-A concentration [13]. In addition, elevated fetuin-A level in obesity is significantly reduced during exercise- and diet-induced weight loss [4]. However, it is not known whether improving lipid metabolism disorder is associated with ameliorating elevated fetuin-A level or reversing increased urine albumin excretion.

Therefore, this study investigated whether high circulating fetuin-A is associated with increased urine albumin excretion in diet-induced obese rats and examined the potential mechanism associating fetuin-A with microalbuminuria. We also studied whether the increased urine albumin excretion induced by higher serum fetuin-A levels was modified by improving hepatic steatosis and lipid metabolism disorder.

\section{Methods}

2.1. Animal Studies. 4-week-old male Wistar rats were purchased from the experimental animal center of west China, Sichuan University, China. The animal protocol was approved by the local ethics committee for animal studies and animals were handled according to "Principles of laboratory animal care". All rats were housed under controlled temperature $\left(20 \pm 2^{\circ} \mathrm{C}\right)$, humidity (50\%), and light conditions (12-h light-dark cycle), and water was freely available. The rats were randomly divided into three groups: the control group $(n=$ 8 ) was fed with normal chow (320 kcal/100 g/day), the obesity group $(n=8)$ was fed with high fat chow $(586 \mathrm{kcal} / 100 \mathrm{~g} / \mathrm{day})$ and the fenofibrate group $(n=8)$ was fed with high fat chow plus fenofibrate $(100 \mathrm{mg} / \mathrm{kg} / \mathrm{d})$. At the end of 24 weeks the rats were sacrificed and the liver was harvested. Liver lobes were weighed and processed for liver triglycerides (LTG), protein, and histological analysis. The blood was collected and serum samples were stored and kept frozen at $-80^{\circ} \mathrm{C}$ for analyzing the levels of alanine aminotransferase (ALT), aspartate aminotransferase (AST), free fatty acids (FFAs), fetuin-A, and adiponectin. Abdominal visceral fat (including perirenal fat, epididymal fat, and mesenteric fat) was collected and weighted.

2.2. Blood Measurements. Plasma glucose was measured by the glucose oxidase method. Serum insulin was assayed by radioimmunoassay (Linco Research, Inc., St Louis, MI, USA). Serum ALT and AST levels were measured by automatic biochemical analysis meter (Beckman, USA). Serum FFA concentrations were assayed by the colorimetric method with serum FFAs kit (E1001, Applygen Technologies Inc., Beijing, China). Serum fetuin-A and adiponectin levels were measured by an ELISA technique using the rat fetuin-A and adiponectin kits (E90178Ra and E90605Ra, resp., Uscn Life Science Inc., China), according to the manufacturer-supplied instructions.
2.3. Analysis of Liver Triglycerides (LTG). Frozen liver tissues were used for analyzing LTG using the tissue TG kit (E1013, Applygen Technologies Inc., Beijing, China). The liver was weighted and put into a manual glass homogenizer with lysis buffer $(20 \mathrm{uL} / \mathrm{mg})$, which was heated and then centrifuged to obtain supernatant for future enzymatic assays, according to the manufacturer-supplied instructions.

2.4. Liver and Kidney Histological Examination. Hepatic and renal cortex tissues were excised from each rat, fixed in $10 \%$ formalin, and embedded in paraffin wax. Paraffin-embedded tissues were sectioned and stained with hematoxylin-eosin (H\&E).

2.5. Liver $N F-\kappa B$ p65 Immunohistochemical Staining. Sections of formalin-fixed, paraffin-embedded tissue were stained by means of an anti-rabbit SP kit (SP-9001, Zhong Shan Inc., Beijing, China). The specimens were incubated with rabbit anti-rat polyclonal NF- $\kappa$ B (p65) antibody ( 1 : 200 dilution; sc-101748, Santa Cruz Biotechnology, Inc.) overnight at $4^{\circ} \mathrm{C}$. The procedure was performed essentially as described [14]. Semiquantitative analysis of NF- $\kappa$ B p65 expression in the liver used image analysis techniques (Image-Pro Plus software 6.0) to determine the gray scale value.

2.6. Measurement of Urine Albumin Excretion. Urine was collected in a metabolic cage at the end of 24 weeks. Urinary albumin was measured using a radioimmunoassay kit (China Institute of Atomic Energy, Beijing, China). Urinary creatinine concentration was measured by the Jaffe method. Urine albumin excretion was defined as urine albumin-tocreatinine ratio (ACR).

2.7. Statistical Analysis. Statistical analysis was performed by the statistical software package SPSS 17.0 for Microsoft windows (SPSS Inc). Data were expressed as means \pm standard deviation. For three group comparisons, data were analyzed by ANOVA. Statistical comparison among two groups was performed with the student's $t$-test. Pearson correlation coefficient " $r$ " was used to measure the relationship between two variables. $P$ values less than 0.05 were considered statistically significant.

\section{Results}

Body weight increased $32.8 \%$ in obese rats in comparison to controls, and visceral fat increased 3.67 times in the obesity group compared to the control group. Compared with controls, obese rats had significantly higher visceral fat and weight $(P<0.01)$. In the fenofibrate group, body weight was duplicated (with some slight differences) compared to the obesity group, but visceral fat decreased $25.7 \%$ in the fenofibrate group when compared to the obesity group; visceral fat and weight were significantly decreased in the fenofibrate group in comparison to the obesity group (Table 1). 


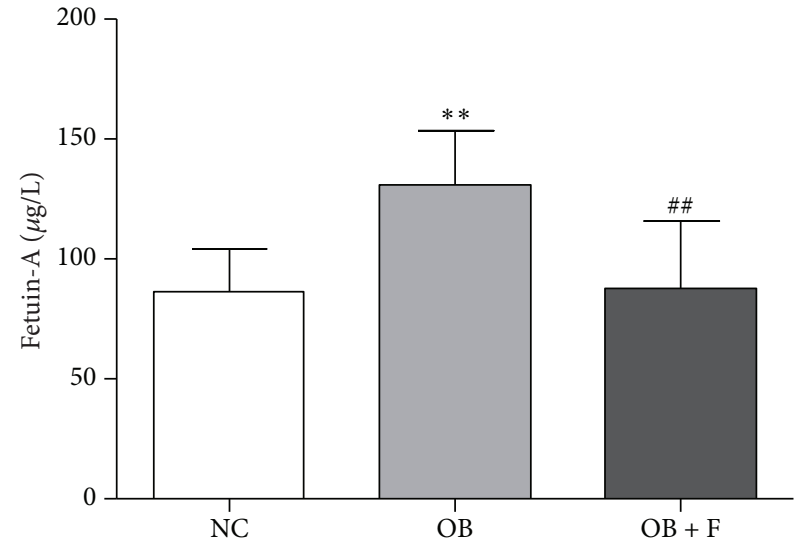

(a)

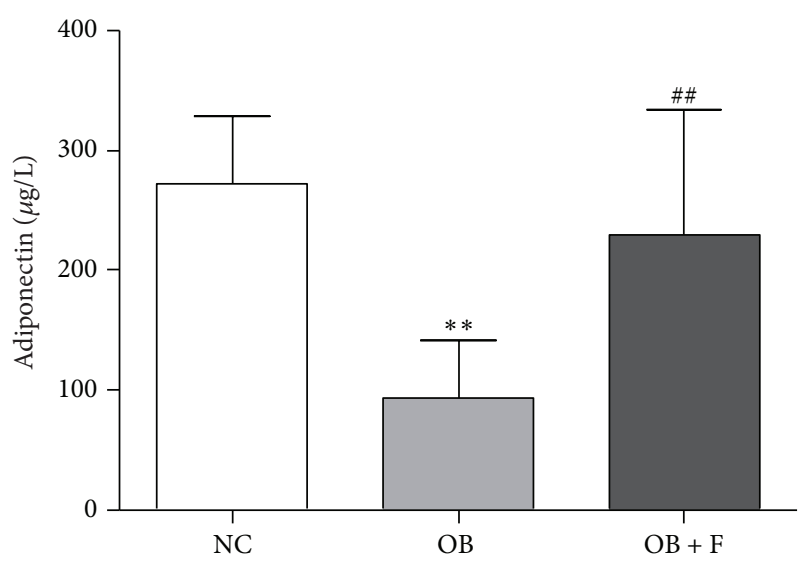

(b)

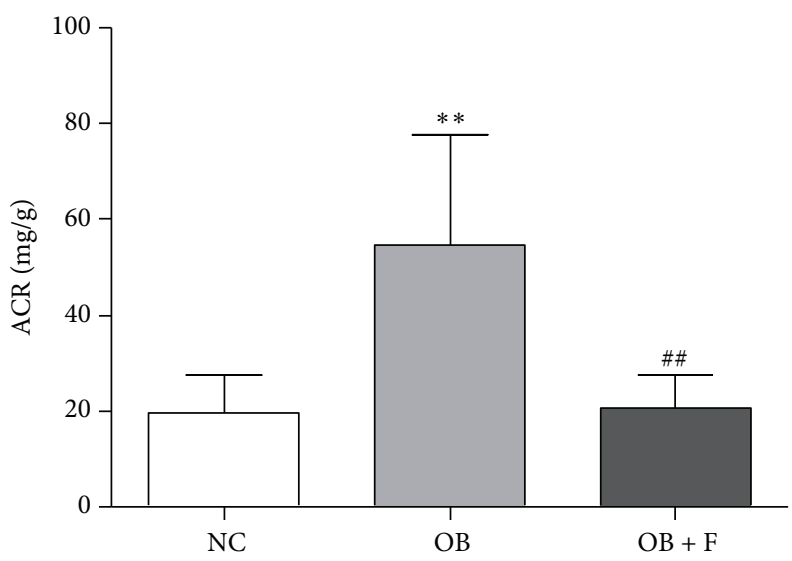

(c)

FIGURE 1: Representative parameters of rats in three groups: levels of fetuin-A (a), adiponectin (b), and ACR (c) in each group. Data was represented by means $\pm \mathrm{SD}(n=8) .{ }^{* *} P<0.01$ versus control group; ${ }^{\# \#} P<0.01$ versus obesity group; $(\mathrm{NC}=$ control group, $\mathrm{OB}=\mathrm{obesity}$ group, $\mathrm{OB}+\mathrm{F}$ = fenofribate group).

TABLE 1: Biometric parameters of rats in the studied groups $(n=8)$.

\begin{tabular}{|c|c|c|c|}
\hline Group & Body weight $(\mathrm{g})$ & Visceral fat (g) & Visceral fat/weight $\left(10^{-3}\right)$ \\
\hline Control group & $521.3 \pm 35.2$ & $14.93 \pm 1.68$ & $28.58 \pm 1.86$ \\
\hline Obesity group & $692.3 \pm 93.9^{* *}$ & $54.90 \pm 14.18^{* *}$ & $73.85 \pm 18.76^{* *}$ \\
\hline Fenofibrate group & $657.4 \pm 56.9^{* *}$ & $40.78 \pm 5.31^{\# \# * *}$ & $55.71 \pm 8.47^{\# \# * *}$ \\
\hline
\end{tabular}

Data are shown as mean $\pm \mathrm{SD} .{ }^{* *} P<0.01$ versus control group. ${ }^{\# \#} P<0.01$ versus obesity group.

There was a significantly increased level of serum insulin, free fatty acids (FFAs), ALT, AST, and LTG in obese rats compared to controls $(P<0.05$, Table 2$)$. A significant decrease in most measurements was detected when fenofibrate was compared to the obesity group $(P<0.05)$, except for LTG content (Table 2). Although the LTG content was apparently decreased in the fenofibrate group, no significant difference was found between the fenofibrate and obesity groups. The data show that the degree of variability was high in obesity group, which might cause the above phenomenon.

There was a significant increase in serum fetuin-A levels in obese rats compared to controls $(130.88 \pm 22.41$ versus $86.24 \pm 17.97 \mu \mathrm{g} / \mathrm{L}, P=0.001)$. A significant decrease was detected in the fenofibrate group when compared to the obesity group $(87.94 \pm 27.7$ versus $130.88 \pm 22.41 \mu \mathrm{g} / \mathrm{L}, P<$ 0.01 ). Serum adiponectin levels were significantly reduced in obese rats in comparison to controls $(93.69 \pm 47.45$ versus $272.27 \pm 56.71 \mu \mathrm{g} / \mathrm{L}, P<0.001)$. A significant increase was found when the fenofibrate group was compared to the obesity group $(228.70 \pm 104.4$ versus $93.69 \pm 47.45 \mu \mathrm{g} / \mathrm{L}$, $P<0.01)$. Urinary albumin/creatinine ratio (ACR) was significantly increased in obese rats accompanied by higher fetuin-A levels and lower adiponectin levels compared to controls $(54.47 \pm 23.26$ versus $19.62 \pm 7.81 \mathrm{mg} / \mathrm{g}, P<0.01)$. The decrease was significant when comparing the fenofibrate group to the obesity group $(20.68 \pm 6.67$ versus $54.47 \pm$ $23.26 \mathrm{mg} / \mathrm{g}, P<0.01)$. Data are shown as mean $\pm \mathrm{SD}$ (Figure 1). 


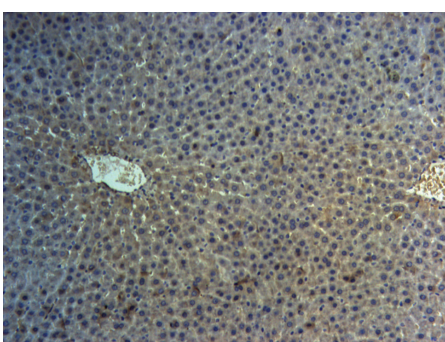

(a)

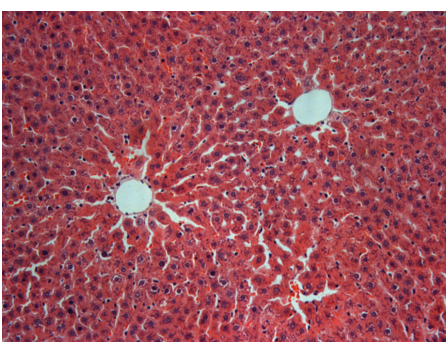

(d)

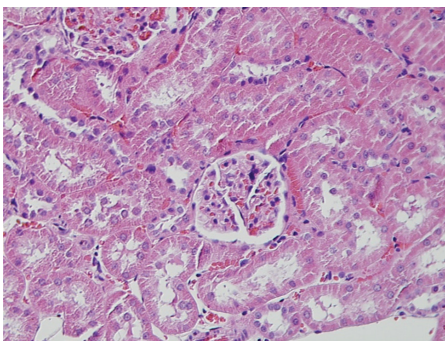

(g)

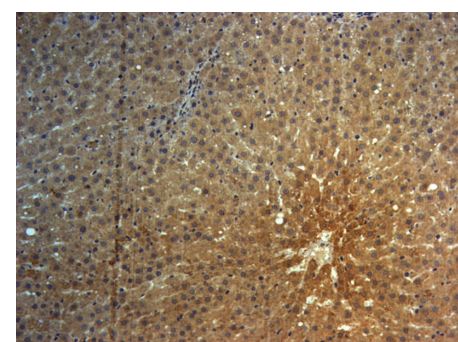

(b)

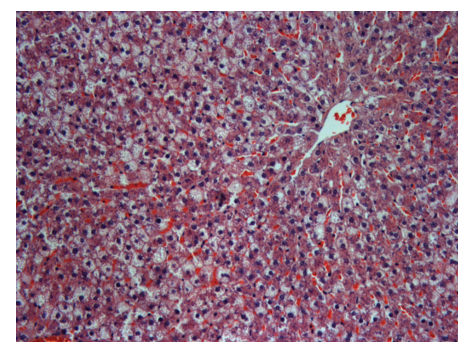

(e)

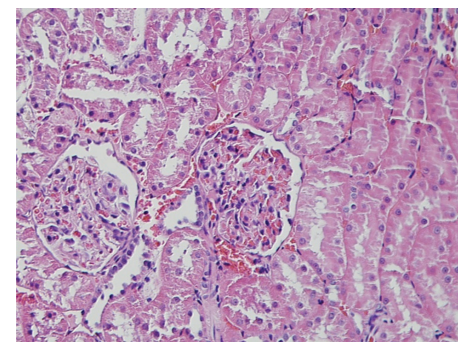

(h)

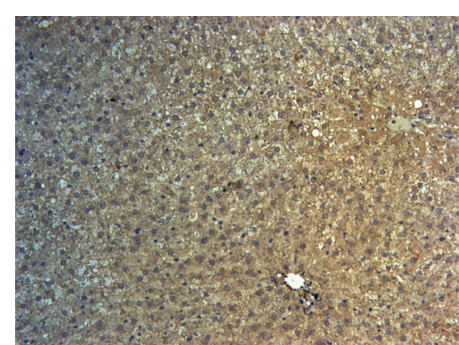

(c)

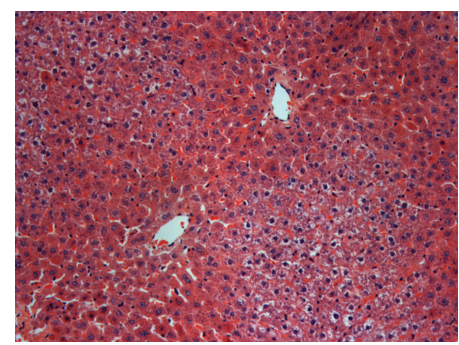

(f)

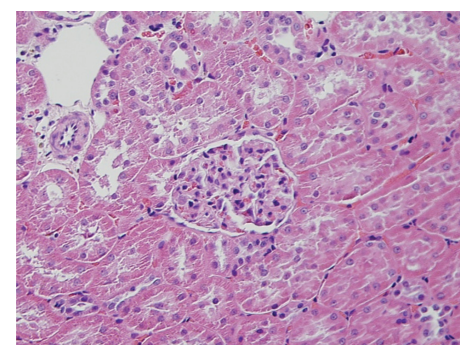

(i)

FIGURE 2: Immunohistochemical staining for NF- $\kappa$ B p65 in the liver in control group (a), obesity group (b), and fenofibrate group (c), respectively. Liver H\&E stain from control group (d), obesity group (e), and fenofribate group (f), respectively. Kidney H\&E stain from control group (g), obesity group (h), and fenofibrate group (i), respectively. Original magnification $\times 200$; $(\mathrm{NC}=$ control group, $\mathrm{OB}=\mathrm{obesity}$ group, and $\mathrm{OB}+\mathrm{F}=$ fenofribate group).

TABLE 2: Blood parameters of rats in three groups $(n=8)$.

\begin{tabular}{|c|c|c|c|c|c|c|}
\hline Group & $\mathrm{FBG}(\mathrm{mmol} / \mathrm{L})$ & FINS (mIU/L) & $\operatorname{ALT}(\mathrm{IU} / \mathrm{L})$ & AST (IU/L) & FFAs (mmol/L) & LTG $(\mu \mathrm{mol} / \mathrm{g})$ \\
\hline Control group & $5.7 \pm 0.4$ & $0.52 \pm 0.19$ & $48.5 \pm 13.7$ & $124 \pm 32.4$ & $0.37 \pm 0.12$ & $110.9 \pm 49.46$ \\
\hline Obesity group & $6.0 \pm 0.4$ & $1.62 \pm 0.24^{*}$ & $83.6 \pm 39.5^{*}$ & $222 \pm 24.3^{*}$ & $1.45 \pm 0.33^{*}$ & $258.8 \pm 127.6^{*}$ \\
\hline Fenofibrate group & $5.9 \pm 0.5$ & $0.89 \pm 0.24^{\# *}$ & $51.5 \pm 12.6^{\#}$ & $108 \pm 13.8^{\#}$ & $0.78 \pm 0.11^{\# *}$ & $192.4 \pm 77.9^{*}$ \\
\hline
\end{tabular}

Data are shown as mean \pm SD. ${ }^{*} P<0.05$ versus control group; ${ }^{\#} P<0.05$ versus obesity group. FBG: fasting blood glucose, FINS: fasting insulin, ALT: Alanine aminotransferase, AST: Aspartate aminotransferase, FFAs: free fatty acid, LTG: liver triglyceride.

Positive NF- $\kappa$ B staining was located in the cytoplasm or nucleus of the liver cells. The NF- $\kappa \mathrm{B}$ expression in the liver was apparently increased in the obese rats (Figure 2(b)) while little NF- $\kappa \mathrm{B}$ expression in the liver was observed in normal rats (Figure 2(a)). After fenofibrate intervention, the increase in NF- $\kappa$ B expression observed in the liver of obese rats was suppressed (Figure 2(c)). Semiquantitative analysis of NF- $\kappa$ B expression in the liver by image analysis techniques showed that there was a $26.3 \%$ increase in obese rats compared to controls $(0.385 \pm 0.031$ versus $0.306 \pm 0.028, P=0.001)$ and an $18.9 \%$ decrease in the fenofibrate group compared to the obesity group $(0.313 \pm 0.045$ versus $0.385 \pm 0.031$, $P<0.05)$. HE-stained liver from diet-induced obese rats revealed a significantly increased steatosis when compared to controls, which was significantly improved after fenofibrate intervention (Figures 2(d)-2(f)). HE-stained kidney revealed marked glomerular enlargement and increased interstitial inflammatory cells (Figures 2(g)-2(i)). However, lowering circulating and renal FFA levels by fenofibrate intervention reduced the glomerular hypertrophy.

Serum FFA concentration and LTG levels were increased in diet-induced obese rats and accompanied by higher serum fetuin-A levels and higher ACR. However, after fenofibrate intervention, lipid metabolism was improved, and simultaneously serum fetuin-A levels were significantly decreased and urine albumin secretion was significantly reduced. 
A significant positive correlation was found between serum fetuin-A levels and ACR $(r=0.602, P=0.002)$. Furthermore, a significant positive correlation was detected between ACR and each of these proteins: serum FFAs, serum ALT, serum AST, and LTG $(r=0.839, P<0.001 ; r=0.727$, $P<0.001 ; r=0.742, P<0.001 ; r=0.715, P<0.001$, resp.). In addition, there was a significant negative correlation between serum adiponectin level and ACR $(r=-0.653$, $P=0.001$; Figure 3 ).

In addition, there was a significant positive correlation between serum fetuin-A and each of these proteins: serum ALT, AST levels, serum FFAs, and LTG content $(r=0.509$, $P<0.05 ; r=0.637, P<0.01 ; r=0.590, P<0.01$; $r=0.428, P<0.05$, resp.). A strong inverse correlation was found between serum fetuin- $A$ and serum adiponectin $(r=-0.537, P<0.01$; Figure 4$)$.

We divided all parameters into tertiles based on ACR: lower, middle, and upper tertiles. Increasing tertiles of ACR were associated with increasing levels of fetuin-A, adiponectin, and NF- $\kappa \mathrm{B}$ (Table 3 ). In addition, there was an obvious increase in serum ALT, AST, and FFAs with increasing tertiles of ACR (Table 3). Although there was no significant difference in LTG content, an apparent increase was found accompanied by increasing tertiles of ACR.

\section{Discussion}

Chronic kidney disease (CKD) is defined by the presence of a marker of kidney damage, such as proteinuria or a decreased glomerular filtration rate for three or more months [2]. CKD has emerged as a growing public health problem worldwide. Microalbuminuria is an early marker of chronic kidney injury, and several cross-sectional studies have noted an association between metabolic syndrome and microalbuminuria in individuals with and without diabetes [15-17]. Ix et al. has documented that higher fetuin-A human concentrations are strongly associated with MetS and an atherogenic lipid profile [6]. Increasing quartiles of fetuin-A are linearly associated with the number of MetS components and increased serum triglyceride concentrations [6]. Additionally, fetuin-A levels strongly, and independently from other important parameters were found to be associated with hyperglycemia [18]. Fetuin-A has been studied as an important circulating inhibitor of ectopic calcium deposition in the renal field, and fetuin-A deficiency in humans was associated with vascular calcification and mortality in patients on hemodialysis [19]. Fetuin-A might, however, have other functions; fetuin-A is an important promoter of insulin resistance and exerts proinflammation effects. This led us to investigate whether higher serum fetuin- $\mathrm{A}$ is associated with abnormal albuminuria.

In the current study, serum fetuin-A levels and microalbuminuria were measured in rats of three different intervention groups to clarify their potential relationship. We are able to show that there was a strong, positive correlation between serum fetuin-A levels and abnormal albuminuria, as reported previously by $\mathrm{Li}$ et al. [20]. The results showed a significant increase in serum fetuin-A levels in obese rats compared to controls. Our study proved that there is a significant positive correlation between serum fetuin-A level and serum ALT, AST level, serum FFA concentration, and LTG content. These results are in accordance with the previous studies that serum fetuin-A levels are elevated in NAFLD $[4,5,21]$. Yilmaz et al. found that serum fetuinA levels are significantly higher in patients with biopsyproven NAFLD and may serve as a biochemical marker of fibrosis in NAFLD patients [21]. However, after diet- and exercise- or surgery-induced weight loss, the homeostatic model assessment (HOMA) index and fetuin-A decreased, and a significant decrease in the prevalence of NAFLD was found $[4,22]$. In addition, a cross-sectional and longitudinal study reported that serum fetuin-A levels were elevated in subjected with fat accumulation in the liver, and a decrease in liver fat was accompanied by a decrease in serum fetuinA concentrations [13]. These findings raise the possibility that fetuin-A may be a new promising link between obesity and its comorbidities. Simultaneously, a significant increase was detected in ACR in obese rats compared to controls. This increase reflects renal impairment and suggests the presence of CKD in obese rats with higher serum fetuin-A levels. A number of studies have shown a positive correlation between obesity and microalbuminuria [23, 24], and that obesity is an independent risk factor for the development of microalbuminuria [25], which is also associated with the MetS and its different components. The prevalence of NAFLD has been estimated to be approximate $90 \%$ in obesity [26]; both NAFLD and dyslipidemia contribute to the rate of progression of renal disease $[1,24]$. Therefore, higher serum fetuin-A is a potential risk factor in diet-induced obese rats and contributes to the prevalence of abnormal albuminuria.

The previous studies have reported that a negative correlation was found between adiponectin and microalbuminuria in obese patients $[12,23]$. Fetuin-A is deemed to repress the production of adiponectin [11]. Based on these findings, we further investigated the association of fetuinA with adiponectin to clarify the potential mechanism that fetuin-A induced the increased urine albumin excretion. We found that serum adiponectin levels were significantly decreased in obese rats in comparison to controls, and our results revealed a strong inverse correlation between serum fetuin- $A$ and serum adiponectin, and a significant inverse correlation between ACR and serum adiponectin. Adiponectin, an adipocyte-derived hormone, has antiinflammatory properties by modulating multiple signaling pathways and exerts largely beneficial effects to improve insulin sensitivity. The decreased level of serum adiponectin represents an independent risk factor for NAFLD [27]. It is well known that chronic low-grade inflammation is a common feature of progression of NAFLD. The results of the present study are in accordance with previous studies $[28,29]$, showing that there was a higher levels of an inflammatory marker, as evidenced by the significant increase in liver NF- $\kappa \mathrm{B}$ expression, in obese rats compared to controls. These results are in accordance with a report that FFAs enhanced fetuin-A secretion commensurate with over-expression and activity of NF- $\kappa \mathrm{B}$ [30]. Another study demonstrated that fetuin-A acted as an endogenous ligand of TLR4, played a key role in FFA-induced 


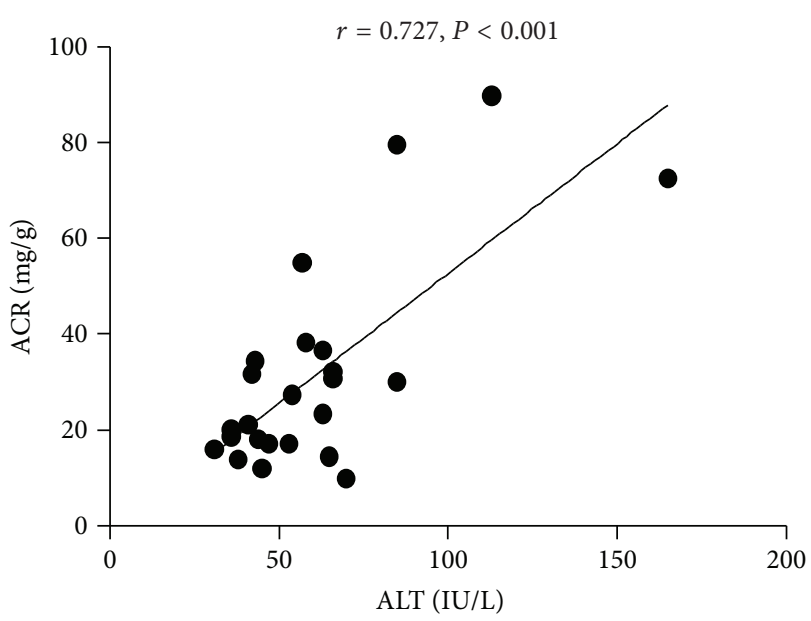

(a)

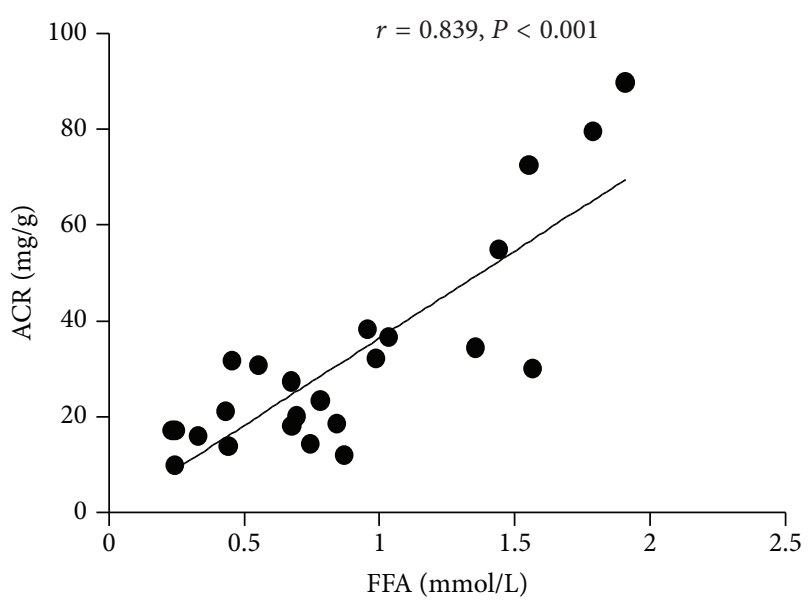

(c)

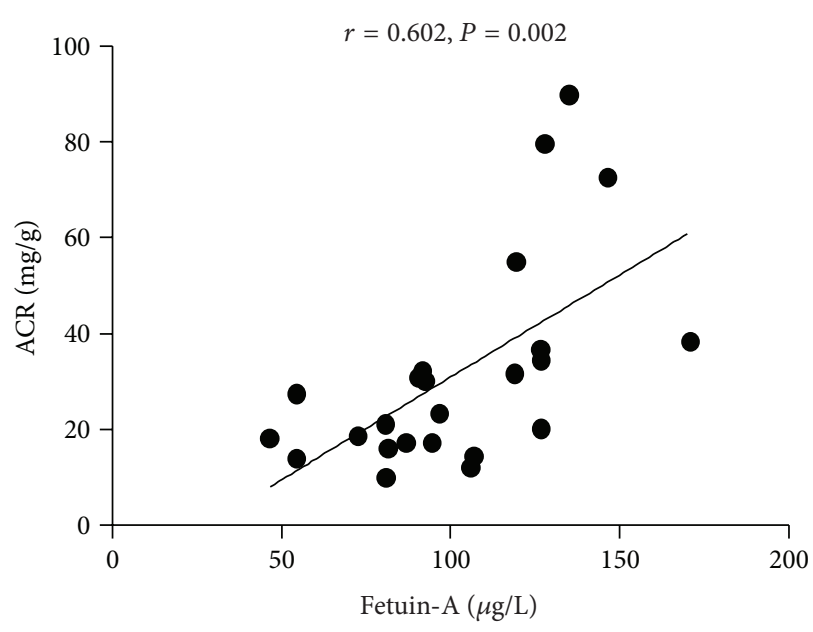

(e)

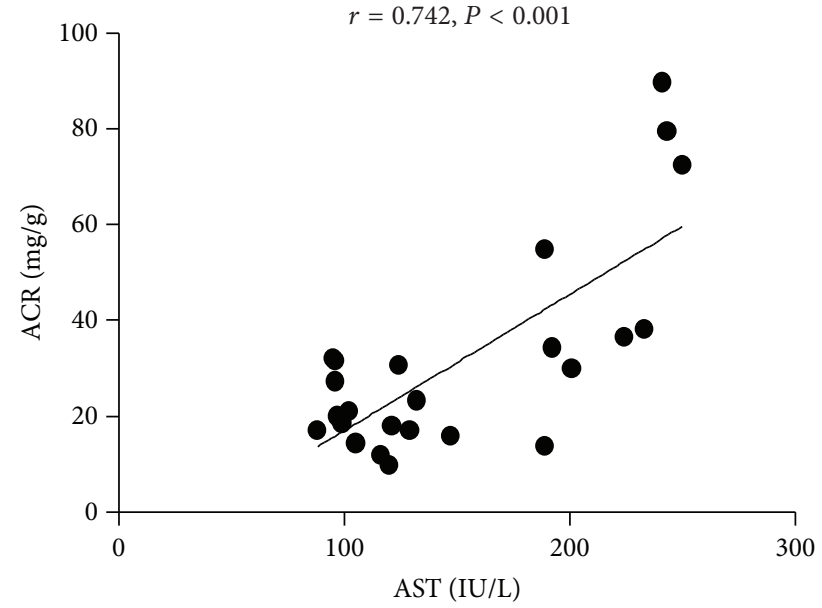

(b)

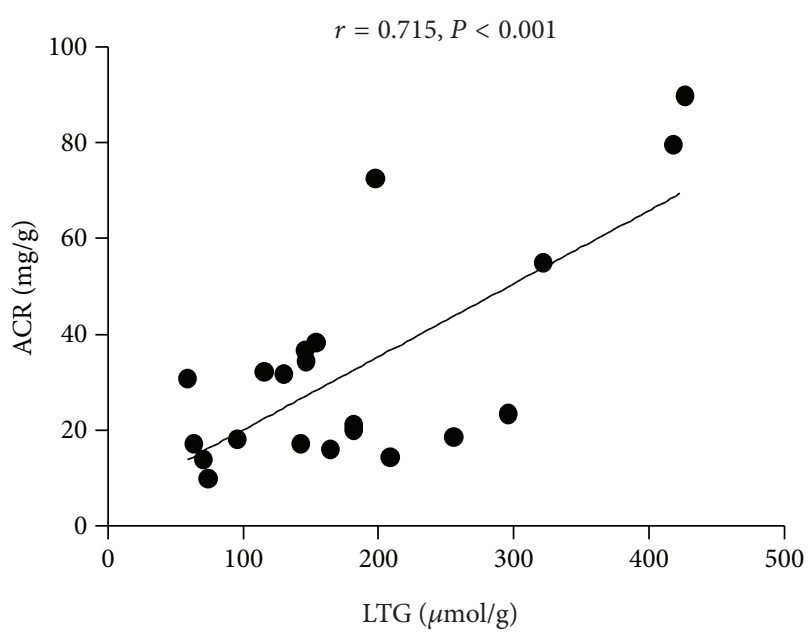

(d)

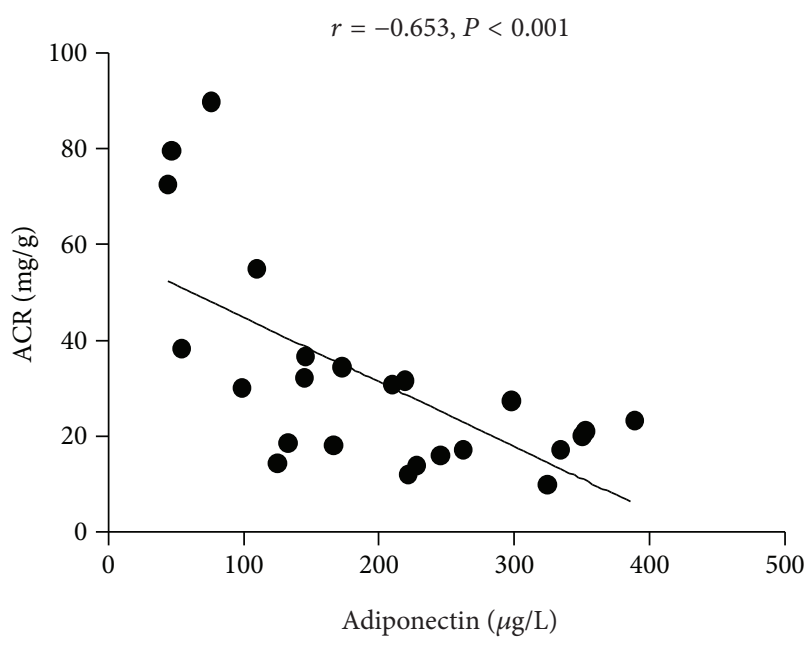

(f)

FIGURE 3: Correlation of serum ALT and AST, serum FFAs, LTG, serum fetuin-A, and adiponectin with the level of ACR; data are from three groups. 


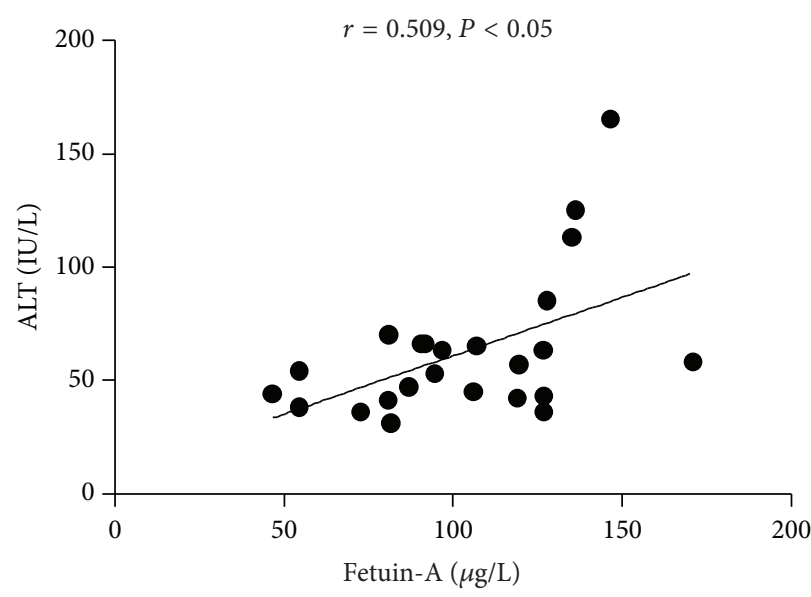

(a)

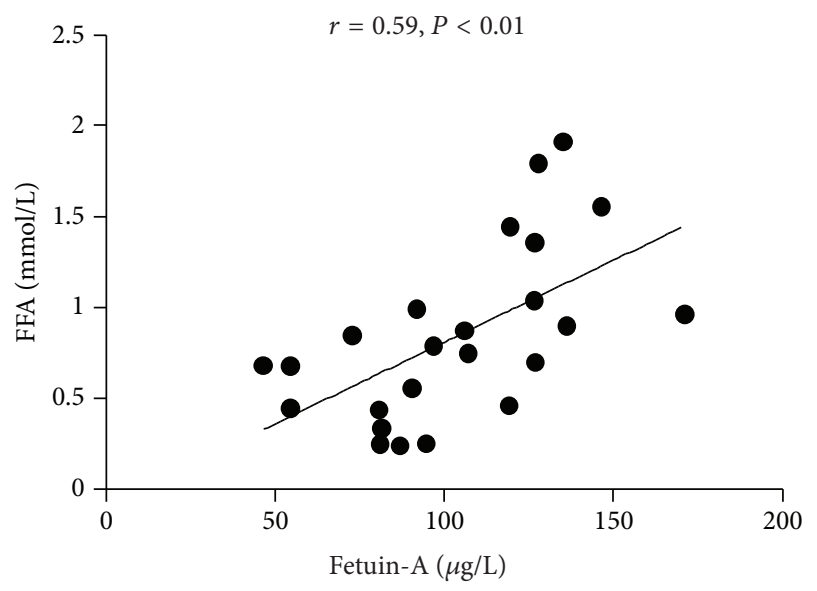

(c)

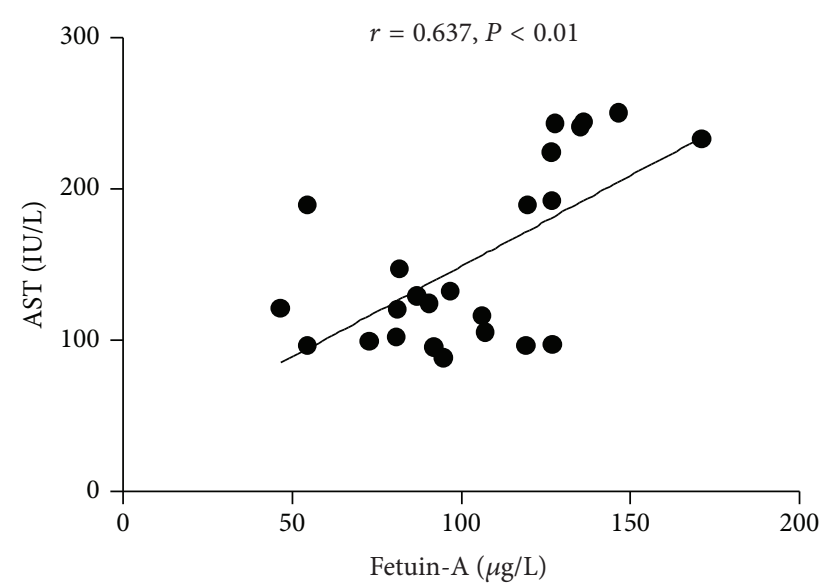

(b)

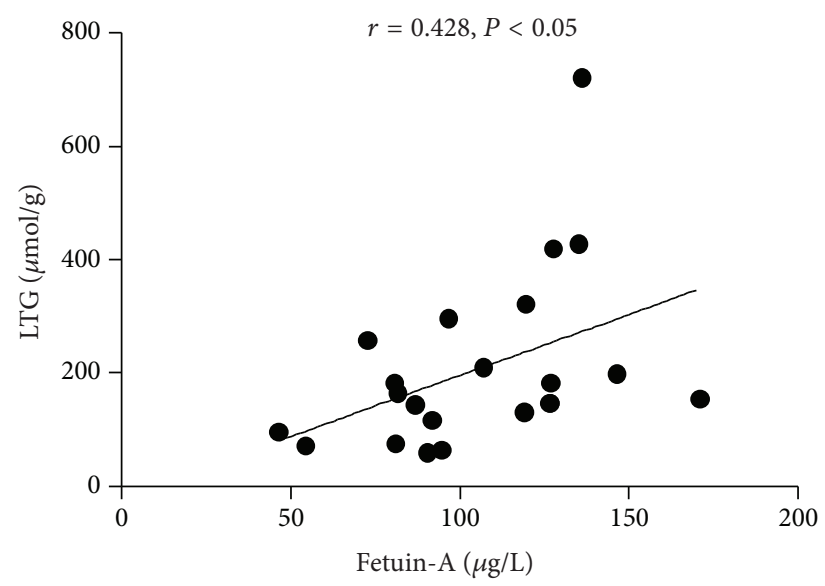

(d)

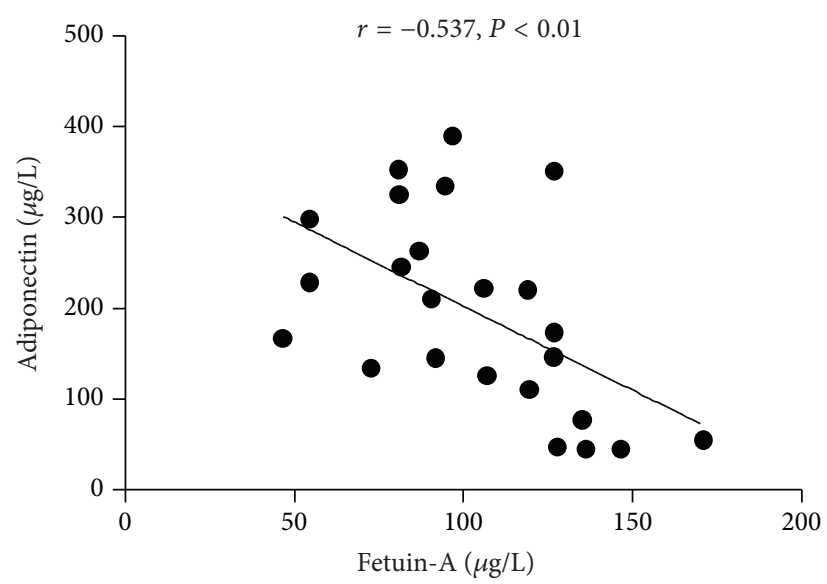

(e)

FIGURE 4: Correlation of serum ALT and AST, serum FFAs, LTG, and serum adiponectin with the level of serum fetuin-A; data are from three groups.

proinflammatory cytokine expression, and promoted lipidinduced insulin resistance [31]. Moreover, the fatty liver itself can exacerbate insulin resistance, thus increasing the risk of renal impairment [32], and several proinflammatory factors from steatosis and inflamed liver can further exacerbate kidney dysfunction [33]. These findings are in accordance with the current study showing that increasing tertiles of
ACR were related with a gradual increase in liver NF$\kappa \mathrm{B}$ expression. These findings provide evidence that higher fetuin-A levels induce increased urine albumin excretion by a mechanism related with repressing adiponectin and triggering inflammation.

Several studies have demonstrated that lifestyle intervention or surgery-induced weight loss leads to a significant 
TABLE 3: Parameters of rats by ACR tertiles.

\begin{tabular}{|c|c|c|c|c|}
\hline & Lower tertile $14.7 \pm 2.9(\mathrm{mg} / \mathrm{g})$ & Middle tertile $25.3 \pm 5.2(\mathrm{mg} / \mathrm{g})$ & Upper tertile $54.7 \pm 22.9(\mathrm{mg} / \mathrm{g})$ & $P$ \\
\hline Fetuin-A $(\mu \mathrm{g} / \mathrm{L})$ & $82.4 \pm 22.1$ & $91.9 \pm 23.6$ & $130.8 \pm 22.6$ & 0.001 \\
\hline $\operatorname{ALT}(\mathrm{IU} / \mathrm{L})$ & $49.1 \pm 13.1$ & $52.9 \pm 17.5$ & $81.3 \pm 40.0$ & $<0.05$ \\
\hline AST (IU/L) & $126.9 \pm 30.4$ & $118.4 \pm 36.2$ & $208.4 \pm 51.2$ & $<0.001$ \\
\hline FFA (mmol/L) & $0.47 \pm 0.25$ & $0.75 \pm 0.36$ & $1.38 \pm 0.37$ & $<0.001$ \\
\hline LTG $(\mu \mathrm{mol} / \mathrm{g})$ & $117.3 \pm 55.7$ & $184.1 \pm 85.1$ & $240.9 \pm 128.5$ & 0.075 \\
\hline Adiponectin $(\mu \mathrm{g} / \mathrm{L})$ & $238.7 \pm 71.4$ & $256.6 \pm 107.6$ & $99.4 \pm 50.8$ & 0.001 \\
\hline $\mathrm{NF}-\kappa \mathrm{B}$ & $0.303 \pm 0.046$ & $0.314 \pm 0.026$ & $0.385 \pm 0.031$ & $<0.05$ \\
\hline
\end{tabular}

Data are shown as mean \pm SD. Data was analyzed by ANOVA. ALT: Alanine aminotransferase, AST: Aspartate aminotransferase, FFA: free fatty acid, LTG: liver triglyceride.

decrease of fetuin-A [4, 22]. In a longitudinal study, a large decrease in liver fat induced by lifestyle intervention was paralleled by a decrease in serum fetuin-A levels [13]. Moreover, Ix et al. found a particularly strong association of human fetuin-A with an atherogenic lipid profile among the components of MetS. However, whether reduced FFA levels could reverse the elevated serum fetuin-A remain unclear. It is known that higher serum FFAs contribute to lipid ectopic deposition in liver, and our findings show that there was a significant increase in FFA concentration and LTG content in obese rats compared to controls. In addition, our study demonstrated that fenofibrate alleviated hepatic steatosis by regulating lipid metabolism (reduced serum FFAs and LTG content); the results also revealed a significant decrease in serum fetuin-A levels, which extends previous findings of decreased fetuin-A levels with improvement of NAFLD after a short-term lifestyle intervention [13]. These findings reflect the reversibility of the increased fetuin-A concentrations. However, in our present study we found that there was no significant difference of LTG levels between the obesity and fenofibrate groups, and that not every LTG level was significantly elevated in the obesity group. This condition indicated that in diet-induced obese rats, fatty liver stayed at a certain stage, as reported by the previous review [7], which is associated with a metabolically benign state. Interestingl, a significant increase was detected in serum adiponectin levels with fenofibrate treatment in comparison to the obesity group, which is in accordance with the previous study that adiponectin was increased after short-term fenofibrate therapy $[13,34]$. Moreover, fenofibrate alleviated hepatic steatosis and decreased serum fetuin-A levels accompanied by improvement in hepatic chronic low-grade inflammation. As the results revealed, a significant decrease was detected in liver NF- $\kappa$ B expression with fenofibrate compared to the obesity group. The steatotic and inflamed liver was alleviated after the fenofibrate intervention in the wake of a reduction in ACR. Therefore, the increased urine albumin excretion is reversible by improving hepatic steatosis.

\section{Conclusions}

The results of the present study indicates that in diet-induced obese rats higher serum fetuin-A levels represent a novel risk factor for the presence of microalbuminuria, and the mechanism may be related with repressing adiponectin production and triggering of chronic low-grade inflammation. Abnormal albuminuria induced by increased fetuin-A levels is reversible by improving hepatic steatosis. Therefore, attention should be paid to obesity or NAFLD with early kidney lesions, and the use of therapeutic agents or lifestyle intervention could be used to prevent NAFLD patients from developing microalbuminuria, thus delaying further renal impairment.

\section{Abbreviations \\ ACR: Urinary albumin/creatinine ratio \\ ALT: Alanine aminotransferase \\ AST: Aspartate aminotransferase \\ CKD: Chronic kidney disease \\ FFAs: Free fatty acid \\ IR: Insulin resistance \\ LTG: Liver triglyceride \\ MAU: Microalbuminuria \\ MetS: Metabolic syndrome \\ NAFLD: Non-alcoholic fatty liver disease \\ T2DM: Type 2 diabetes mellitus.}

\section{Conflict of Interests}

All authors declare that there are no known conflict of interests related with this publication.

\section{Authors' Contribution}

Y. Li wrote the paper and conducted the research; X. Sun conducted the research; Y. Yu reviewed the paper and contributed to the paper.

\section{Acknowledgment}

This work was supported by a National Natural Science Foundation of China Grants (number 81070675). 


\section{References}

[1] Y. Chang, S. Ryu, E. Sung et al., "Nonalcoholic fatty liver disease predicts chronic kidney disease in nonhypertensive and nondiabetic Korean men," Metabolism: Clinical and Experimental, vol. 57, no. 4, pp. 569-576, 2008.

[2] G. Targher, M. Chonchol, G. Zoppini, C. Abaterusso, and E. Bonora, "Risk of chronic kidney disease in patients with non-alcoholic fatty liver disease: is there a link?" Journal of Hepatology, vol. 54, no. 5, pp. 1020-1029, 2011.

[3] S. T. Hwang, Y. K. Cho, J. W. Yun et al., "Impact of nonalcoholic fatty liver disease on microalbuminuria in patients with prediabetes and diabetes," Internal Medicine Journal, vol. 40, no. 6, pp. 437-442, 2010.

[4] T. Reinehr and C. L. Roth, "Fetuin-A and its relation to metabolic syndrome and fatty liver disease in obese children before and after weight loss," Journal of Clinical Endocrinology and Metabolism, vol. 93, no. 11, pp. 4479-4485, 2008.

[5] J. W. Haukeland, T. B. Dahl, A. Yndestad et al., "Fetuin A in nonalcoholic fatty liver disease: in vivo and in vitro studies," European Journal of Endocrinology, vol. 166, no. 3, pp. 503-510, 2012.

[6] J. H. Ix, M. G. Shlipak, V. M. Brandenburg, S. Ali, M. Ketteler, and M. A. Whooley, "Association between human fetuin-A and the metabolic syndrome: data from the heart and soul study," Circulation, vol. 113, no. 14, pp. 1760-1767, 2006.

[7] N. Stefan and H. U. Haring, "The metabolically benign and malignant fatty liver," Diabetes, vol. 60, no. 8, pp. 2011-2017, 2011.

[8] C. Buechler, J. Wanninger, and M. Neumeier, "Adiponectin, a key adipokine in obesity related liver diseases," World Journal of Gastroenterology, vol. 17, no. 23, pp. 2801-2811, 2011.

[9] A. H. Kissebah, G. E. Sonnenberg, J. Myklebust et al., "Quantitative trait loci on chromosomes 3 and 17 influence phenotypes of the metabolic syndrome," Proceedings of the National Academy of Sciences of the United States of America, vol. 97, no. 26, pp. 14478-14483, 2000.

[10] N. Vionnet, E. H. Hani, S. Dupont et al., "Genomewide search for type 2 diabetes-susceptibility genes in French whites: evidence for a novel susceptibility locus for early-onset diabetes on chromosiome 3q27-qter and independent replication of a type 2-diabetes locus on chromosome 1q21-q24," American Journal of Human Genetics, vol. 67, no. 6, pp. 1470-1480, 2000.

[11] A. M. Hennige, H. Staiger, C. Wicke et al., "Fetuin-A induces cytokine expression and suppresses adiponectin production," PLoS ONE, vol. 3, no. 3, article e1765, 2008.

[12] K. Sharma, S. RamachandraRao, G. Qiu et al., "Adiponectin regulates albuminuria and podocyte function in mice," Journal of Clinical Investigation, vol. 118, no. 5, pp. 1645-1656, 2008.

[13] N. Stefan, A. M. Hennige, H. Staiger et al., " $\alpha_{2}$-HeremansSchmid glycoprotein/fetuin-A is associated with insulin resistance and fat accumulation in the liver in humans," Diabetes Care, vol. 29, no. 4, pp. 853-857, 2006.

[14] R. A. DeAngelis, K. Kovalovich, D. E. Cressman, and R. Taub, "Normal liver regeneration in p50/nuclear factor $\kappa \mathrm{B} 1$ knockout mice," Hepatology, vol. 33, no. 4, pp. 915-924, 2001.

[15] F. Bonnet, M. Marre, J. M. Halimi et al., "Waist circumference and the metabolic syndrome predict the development of elevated albuminuria in non-diabetic subjects: the DESIR study," Journal of Hypertension, vol. 24, no. 6, pp. 1157-1163, 2006.

[16] L. Palaniappan, M. Carnethon, and S. P. Fortmann, "Association between microalbuminuria and the metabolic syndrome:
NHANES III," American Journal of Hypertension, vol. 16, no. 11, part 1, pp. 952-958, 2003.

[17] J. Chen, P. Muntner, L. L. Hamm et al., "The metabolic syndrome and chronic kidney disease in U.S. adults," Annals of Internal Medicine, vol. 140, no. 3, pp. 167-I39, 2004.

[18] K. Kantartzis, J. MacHann, F. Schick, A. Fritsche, H. U. Häring, and N. Stefan, "The impact of liver fat vs visceral fat in determining categories of prediabetes," Diabetologia, vol. 53, no. 5, pp. 882-889, 2010.

[19] M. Ketteler, P. Bongartz, R. Westenfeld et al., "Association of low fetuin-A (AHSG) concentrations in serum with cardiovascular mortality in patients on dialysis: a cross-sectional study," Lancet, vol. 361, no. 9360, pp. 827-833, 2003.

[20] M. Li, M. Xu, Y. Bi et al., "Association between higher serum fetuin-A concentrations and abnormal albuminuria in middleaged and elderly Chinese with normal glucose tolerance," Diabetes Care, vol. 33, no. 11, pp. 2462-2464, 2010.

[21] Y. Yilmaz, O. Yonal, R. Kurt et al., "Serum fetuin A/ $\alpha_{2}$ HSglycoprotein levels in patients with non-alcoholic fatty liver disease: relation with liver fibrosis," Annals of Clinical Biochemistry, vol. 47, no. 6, pp. 549-553, 2010.

[22] J. M. Brix, H. Stingl, F. Höllerl, G. H. Schernthaner, H. P. Kopp, and G. Schernthaner, "Elevated fetuin-A concentrations in morbid obesity decrease after dramatic weight loss," Journal of Clinical Endocrinology and Metabolism, vol. 95, no. 11, pp. 4877-4881, 2010.

[23] K. Sharma, "The link between obesity and albuminuria: adiponectin and podocyte dysfunction," Kidney International, vol. 76, no. 2, pp. 145-148, 2009.

[24] A. Savino, P. Pelliccia, F. Chiarelli, and A. Mohn, "Obesityrelated renal injury in childhood," Hormone Research in Paediatrics, vol. 73, no. 5, pp. 303-311, 2010.

[25] M. Pavan, R. Ranganath, A. PChudhari, and M. Shetty, "Obesity as an independent risk factor for the development of microalbuminuria," Nephro-Urology Monthly, vol. 3, no. 4, pp. 276-279, 2011.

[26] G. Marchesini, S. Moscatiello, S. di Domizio, and G. Forlani, "Obesity-associated liver disease," Journal of Clinical Endocrinology and Metabolism, vol. 93, no. 11, supplement 1, pp. s74-s80, 2008.

[27] Y. Wang, Z. Mingyan, K. S. L. Lam, and A. Xu, "Protective roles of adiponectin in obesityrelated fatty liver diseases: mechanisms and therapeutic implications," Arquivos Brasileiros de Endocrinologia e Metabologia, vol. 53, no. 2, pp. 201-212, 2009.

[28] L. A. Videla, G. Tapia, R. Rodrigo et al., "Liver NF- $\kappa$ B and AP1 DNA binding in obese patients," Obesity, vol. 17 , no. 5, pp. 973-979, 2009.

[29] L. Li, L. Chen, L. Hu et al., "Nuclear factor high-mobility group box 1 mediating the activation of toll-like receptor 4 signaling in hepatocytes in the early stage of nonalcoholic fatty liver disease in mice," Hepatology, vol. 54, no. 5, pp. 1620-1630, 2011.

[30] S. Dasgupta, S. Bhattacharya, A. Biswas et al., "NF- $\kappa$ B mediates lipid-induced fetuin-A expression in hepatocytes that impairs adipocyte function effecting insulin resistance," Biochemical Journal, vol. 429, no. 3, pp. 451-462, 2010.

[31] D. Pal, S. Dasgupta, R. Kundu et al., "Fetuin-A acts as an endogenous ligand of TLR4 to promote lipid-induced insulin resistance," Nature Medicine, vol. 18, no. 8, pp. 1279-1285, 2012.

[32] P. A. Sarafidis and L. M. Ruilope, "Insulin resistance, hyperinsulinemia, and renal injury: mechanisms and implications," American Journal of Nephrology, vol. 26, no. 3, pp. 232-244, 2006. 
[33] G. Targher, F. Marra, and G. Marchesini, "Increased risk of cardiovascular disease in non-alcoholic fatty liver disease: causal effect or epiphenomenon?" Diabetologia, vol. 51, no. 11, pp. 1947-1953, 2008.

[34] R. S. Rosenson, "Effect of fenofibrate on adiponectin and inflammatory biomarkers in metabolic syndrome patients," Obesity, vol. 17, no. 3, pp. 504-509, 2009. 


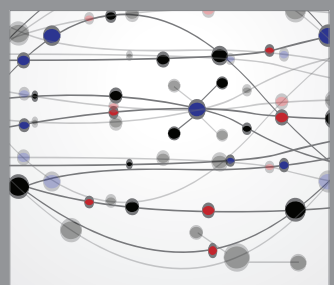

The Scientific World Journal
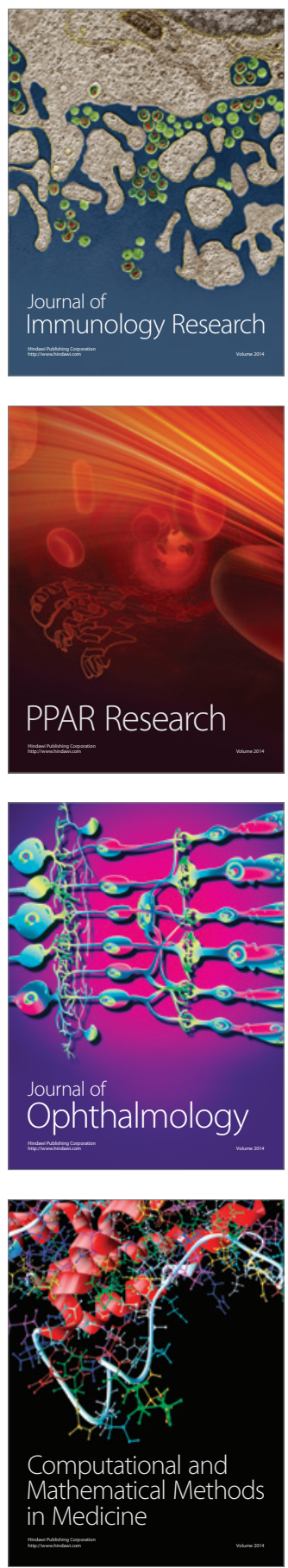

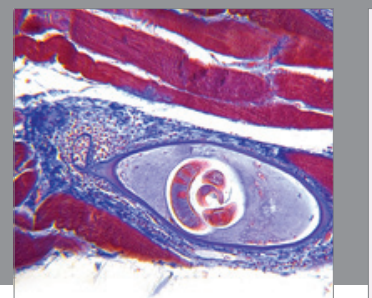

Gastroenterology

Research and Practice
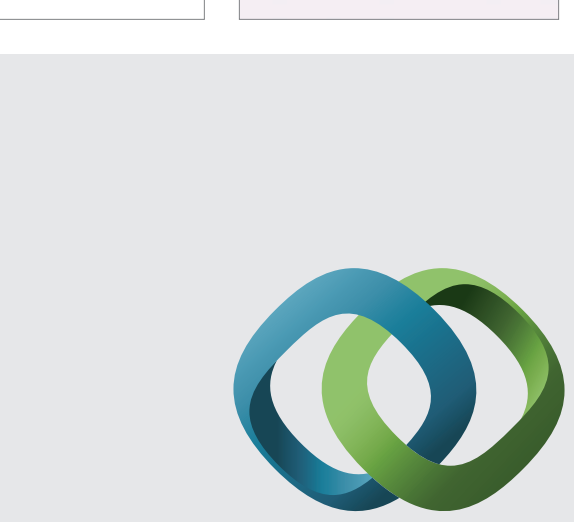

\section{Hindawi}

Submit your manuscripts at

http://www.hindawi.com
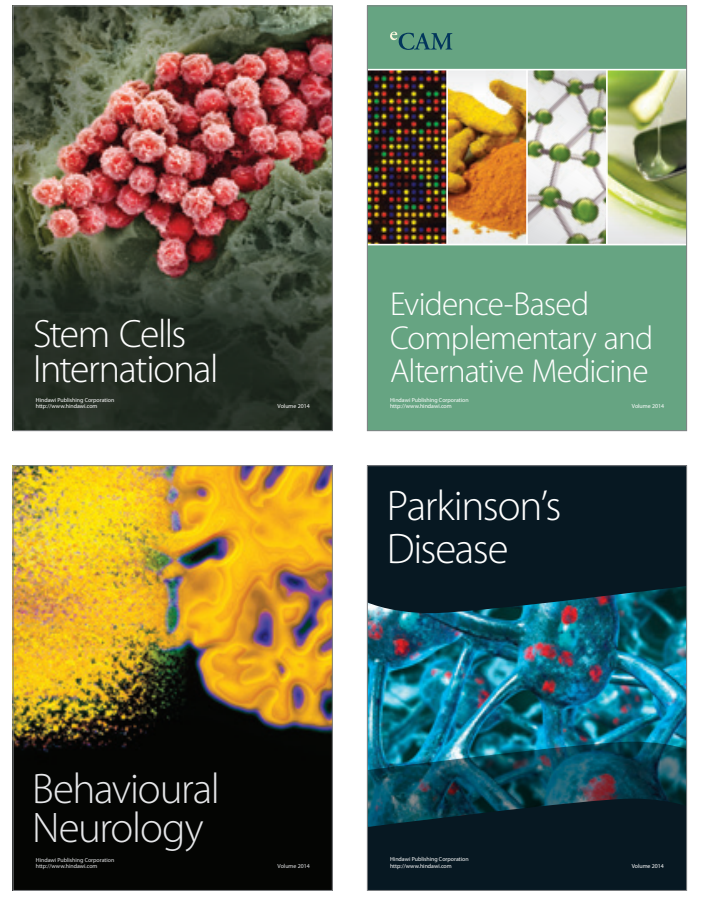
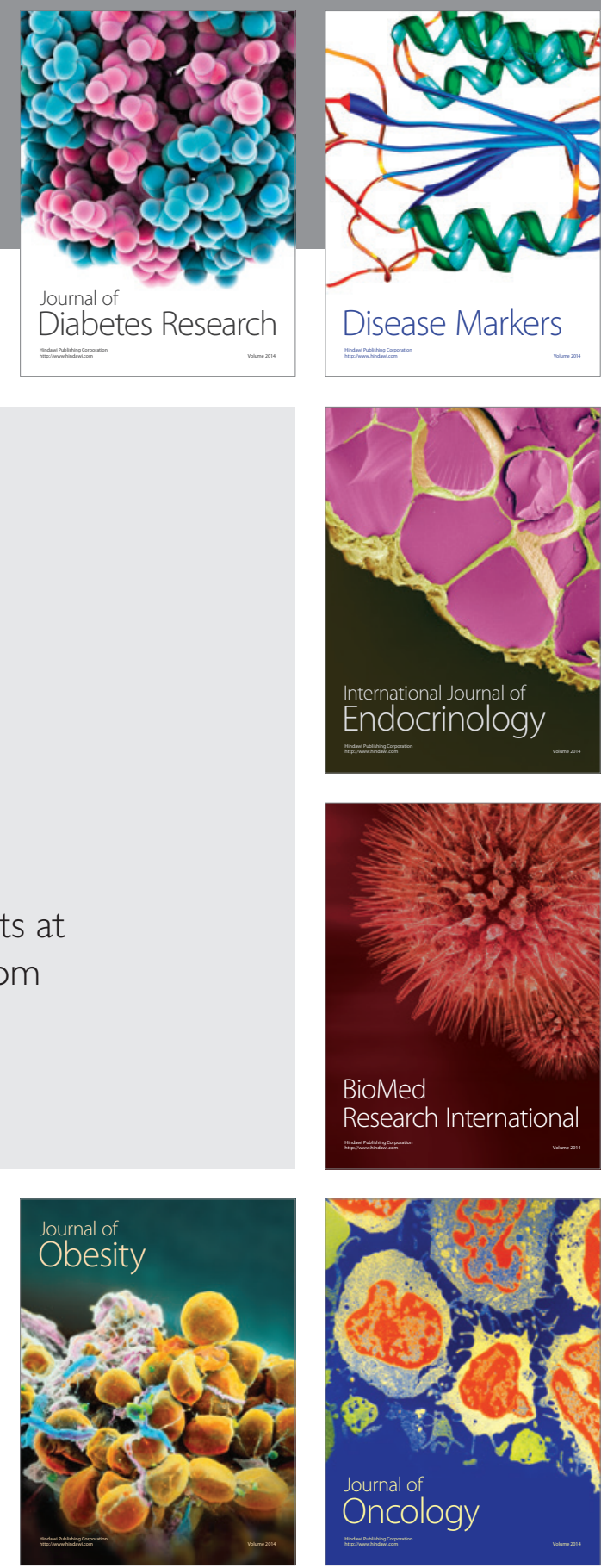

Disease Markers
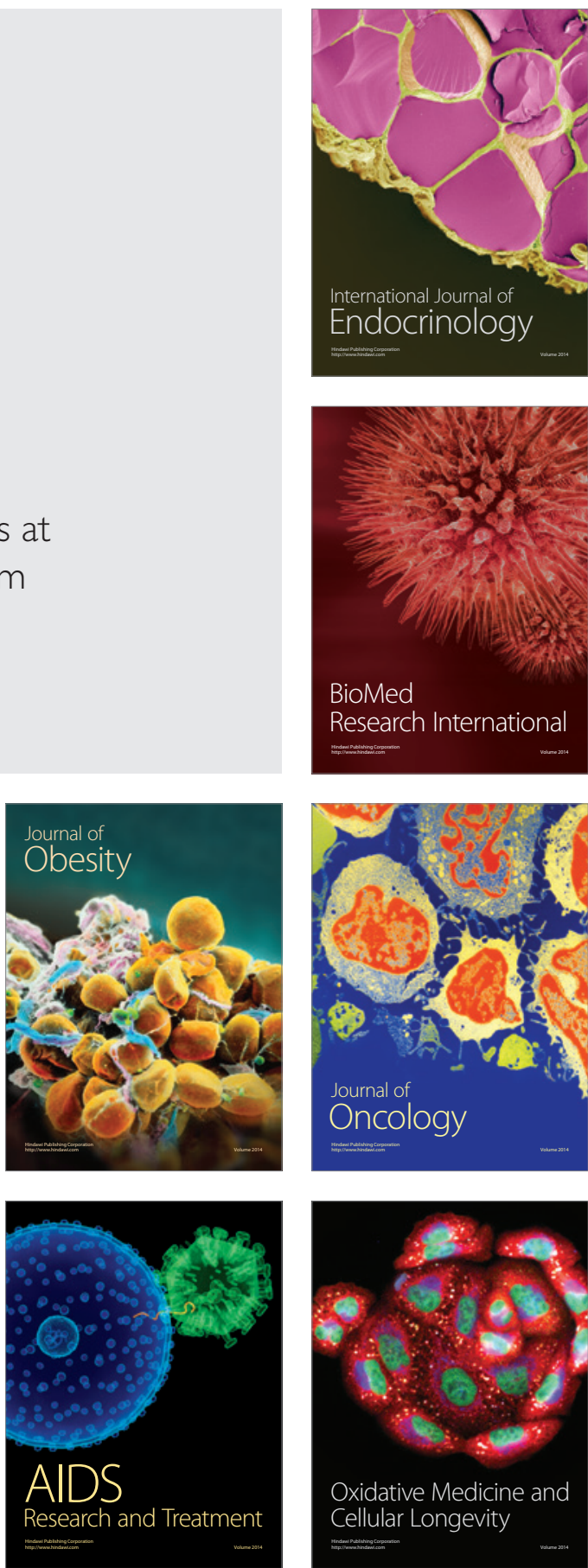